\title{
Two and three nucleon induced photon absorption
}

\author{
E. Oset \\ Departamento de Física Teórica and IFIC, Centro Mixto Universidad de Valencia-CSIC, 46100 Burjassot, Valencia, Spain \\ A. Ramos \\ Departament d'Estructura i Constituents de la Matèria, Universitat de Barcelona, Diagonal 647, 08028 Barcelona, Spain
}

(Received 27 March 1995)

\begin{abstract}
We have investigated the mechanisms leading to two and three body photon absorption in nuclei. At photon energies around the pion production threshold we obtain a fraction of three body absorption of less than $10 \%$ of the total, contradicting previous theoretical claims that it dominates the absorption process. The strength of the three body channel grows smoothly with the photon energy reaching a maximum of about $60 \%$ of the total direct absorption at energies of the photon around $400 \mathrm{MeV}$.
\end{abstract}

PACS number(s): 25.20.Dc

\section{INTRODUCTION}

The subject of how many nucleons are involved in photon absorption in nuclei is bound to follow a similar trend as the same question in pion absorption. This latter topic has attracted much attention experimentally [1] and the findings in medium and heavy nuclei support a picture of pion absorption where the two nucleon mechanism is largely dominant at low pion energies but the three body mechanism becomes more important as the energy increases, reaching a maximum around $T_{\pi}=250 \mathrm{MeV}$ where its strength is about one-half of the total absorption rate. The experimental findings follow closely the theoretical predictions of Ref. [2] calculated up to $T_{\pi}=400 \mathrm{MeV}$. A semiphenomenological evaluation at energies above the $\Delta$ resonance shows the three body absorption playing a smaller role than the two body one [3].

When talking about two and three body absorption one must clarify its meaning. Usually many particles are involved in the process of pion absorption. For instance a pion can undergo quasielastic collisions with other nucleons before it is finally absorbed [4]. On the other hand the nucleons coming from the quasielastic collisions or pion absorption can scatter with other nucleons on their way out. Hence one distinguishes the genuine absorption mechanisms as the onestep processes where the pion is absorbed, leaving apart initial state interaction of the pion or final state interaction of the nucleons. In terms of Feynman diagrams for the process it means that genuine absorption diagrams are those where on shell pions and on shell nucleons do not appear in the intermediate lines of the absorption amplitude. Since the particles involved in the diagrams will be off shell, they are short lived and the process is of relatively short range.

Similarly, when we talk here about genuine (or direct) photon absorption we refer again to mechanisms in which real pions or real nucleons are not present in intermediate states in the absorption amplitude. The contribution of such mechanisms in the region of dominance of the $\Delta$ has been studied in Ref. [5]. In addition to this, one has alternative mechanisms for photon absorption in which a real pion is created through the $(\gamma, \pi)$ reaction and the pion is subsequently absorbed by two or three nucleons. This alternative way of photon absorption is usually addressed as indirect photon absorption and has proved to be very important at energies around the $\Delta$ excitation and above in light [6] and heavy [7] nuclei.

In Ref. [8] it was suggested that the whole strength of photon absorption around the pion production threshold could be due to genuine three nucleon mechanisms in the sense used here. Comparisons of this model with estimates of two body absorption, based upon the empirical quasideuteron model, were made in Ref. [9] and reinforced the idea that photon absorption should be dominated by three body absorption around pion creation threshold. However, calculations of the two body absorption channels at the same energy [5] show that the two body mechanisms provide a reasonable reproduction of the experimental data [10-12].

On the other hand some experimental analyses around the same photon energy in $\left(e, e^{\prime}\right)$ reactions came to suggest that three body absorption might account indeed for a considerable fraction of the total absorption rate [13]. Some other data in light nuclei suggest smaller fractions of three body absorption [14]. The experimental situation is now experiencing a boom and plenty of data are being analyzed, which should shed light on these issues [15].

Meanwhile the idea of Ref. [8] stimulated an interesting work on two nucleon induced $\Lambda$ decay [16] which had repercussions on the neutron to proton induced $\Lambda$ decay ratio, one of the puzzles in $\Lambda$ hypernuclear decay [17]. The idea was caught in Ref. [18], improving on several approximations of Ref. [16], with the result that the two nucleon induced channel represents about $15 \%$ of the one nucleon induced channel, but still can have large repercussions in the experimental analysis trying to extract the ratio of $n$ to $p$ induced $\Lambda$ decay. The steps given in Refs. [16] and [18] have laid the ground for an accurate evaluation of the three nucleon mechanism suggested in Ref. [8] for photon absorption and its comparison with the two nucleon mechanisms studied in Ref. [5]. This is the purpose of the present work. The analogy with the two nucleon and one nucleon induced $\Lambda$ decay in nuclei is apparent and we shall closely follow Refs. [16] and [18] in the evaluation of the absorption rates. 


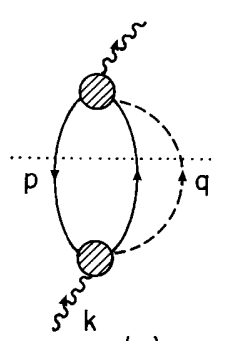

(a)

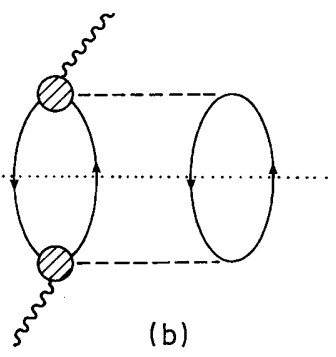

FIG. 1. (a) Photon self-energy diagram obtained by folding the $\gamma N \rightarrow \pi N$ amplitude. The circle indicates the sum of the terms of this amplitude. (b) Photon self-energy diagram obtained from the one in (a) when the pion is allowed to excite a $p h$. The cut in the figure (dotted line) corresponds to two nucleon photon absorption.

The details of the formalism and the approximations used are presented in Secs. II-IV. The results are shown and discussed in Sec. V. Finally, Sec. VI summarizes our conclusions.

\section{GENERAL CONSIDERATIONS ON PHOTON ABSORPTION IN NUCLEI}

The many body approach followed in Refs. [5,7] offers a unified treatment of all the reaction channels in photon nucleus scattering. At photon energies below $500 \mathrm{MeV}$ those channels are essentially $(\gamma, \pi)$ and photon absorption by nucleons. The starting point in Ref. [5] is a model for the elementary $\gamma N \rightarrow \pi N$ reaction, similar to the one of Ref. [19], which contains the pion pole, Kroll-Ruderman term, nucleon direct and crossed terms, and $\Delta$ direct and crossed terms. One can obtain photon self-energy diagrams by folding the terms of the $\gamma N \rightarrow \pi N$ amplitude and summing over occupied states. Such terms are depicted in Fig. 1(a). At higher orders in the nuclear density the pion in Fig. 1(a) is allowed to interact with the medium, exciting a particle hole $(p h)$, for instance, as depicted in Fig. 1(b). Higher order terms in the nuclear density are also considered in Ref. [5]. As an example we show in Fig. 2 terms involving one, two, or three occupied nucleons or, equivalently, terms linear, quadratic, and cubic in the nuclear density approximately.

The total $\gamma$ nuclear reaction cross section [omitting the negligible Compton and coherent $\left(\gamma, \pi^{0}\right)$ channels] is given by [5]

$$
\sigma=-\frac{1}{k} \int d^{3} r \operatorname{Im} \Pi(k, \rho(\vec{r})),
$$

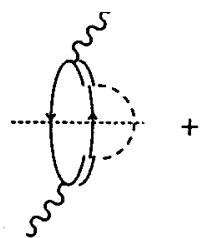

(a)

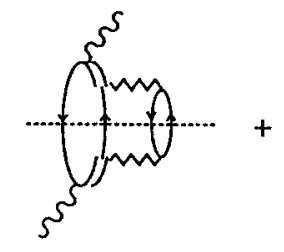

(b)

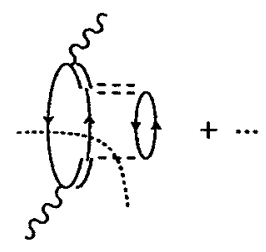

(c)

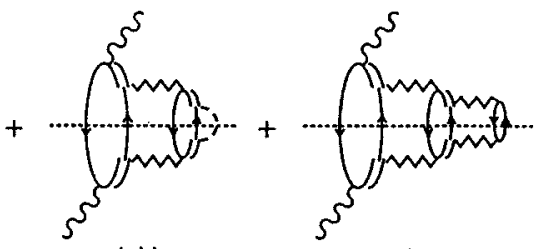

(d) (e)
FIG. 2. Diagrammatic representation of the $\Delta h$ photonuclear excitation piece, including $\Delta$ self-energy corrections. The dotted lines indicate the sources of imaginary part of the photon selfenergy when the internal lines cut by the dotted line are placed on shell. The cut in (a) accounts for the $(\gamma, \pi)$ channel, the one in (b) for $\gamma$ absorption by two nucleons, and the one in (c) for corrections to $(\gamma, \pi)$ when the pion, in the pion exchange included as part of the interaction, is placed on shell. The cut in (d) accounts for $2 p 2 h 1 \pi$ excitation. The cut in (e) accounts for $\gamma$ absorption by three nucleons.

where $\Pi(k, \rho)$ is the self-energy of a photon of momentum $k$ in nuclear matter of density $\rho$. A local density approximation is implicit in Eq. (1) since $\rho$ is substituted by $\rho(\vec{r})$, the local density of the nucleus, and an integral is done over the whole nuclear volume. This approximation was found to be extremely good in Ref. [5], where it was mathematically proved that folding the local self-energy with any finite range of the interaction leads exactly to the same cross section.

The imaginary part of the photon self-energy is obtained from the many body diagrams when the set of intermediate states cut by the dotted lines in Figs. 1 and 2 is placed on shell in the intermediate integrations, a procedure which is easily implemented in terms of Cutkosky rules. In Fig. 2 one can see that the cuts of (a), (c), and (d) lead to the $(\gamma, \pi)$ channel, while (b) leads to $2 N$ photon absorption and (e) to $3 N$ photon absorption.

Let us concentrate in diagram (e) of Fig. 2. The evaluations in Ref. [5] are done omitting the on shell pion in the interaction line (serrated line) closest to the photon in the figure. This is done to obtain the genuine absorption contribution. Assume for a moment that the interaction line corresponds to a pion and the pion is on shell. The process then qualifies as a $(\gamma, \pi)$ step followed by the absorption of the pion by two nucleons, which is what we call indirect photon absorption and is evaluated in Ref. [7]. This latter result looks intuitive but an analytical proof can be found in Ref. [20]. At photon energies where the pion has a kinetic energy of around $100 \mathrm{MeV}$ or more, the propagation of these pions in the nucleus can be done accurately in terms of a Monte Carlo simulation [21] where the real pions move along classical trajectories between collisions or before absorption, with probabilities for these processes calculated with the many body scheme of Ref. [5]. However, if we assume once 


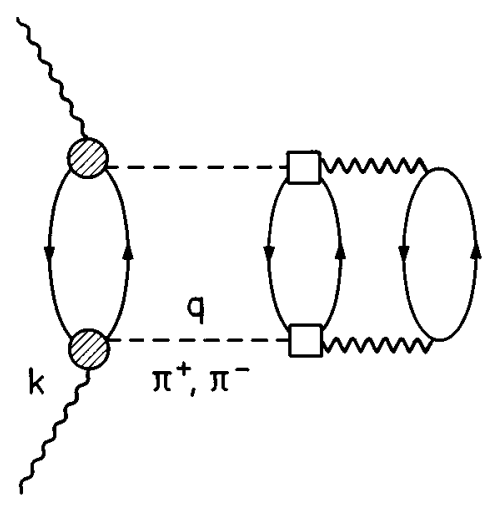

FIG. 3. Photon self-energy diagram accounting for the three body absorption discussed in Ref. [8].

more one pion exchange (OPE) in the same interaction line and go down with the photon energy just below pion production threshold, then the OPE corresponds necessarily to an off shell pion and the mechanism has become a genuine three body absorption. This is so since in the photon absorption diagram [just the lower half of diagram (e) below the dotted line] there are no on shell pion or nucleon intermediate lines. The process involves the absorption of the photon by three nucleons and is a one-step process.

This latter mechanism is the one considered in Ref. [8], although we have used a different language and motivation in order to put the mechanism in a general framework in the light of recent findings in photoabsorption. On the other hand, such a contribution for the $\Delta$ excitation pieces in Fig. 2 is also considered in Refs. [2] and [5], but this is irrelevant for photon absorption around pion threshold because, as shown in Fig. 49 of Ref. [5], photon absorption at these energies is practically all given by the nonresonant terms, led essentially by the Kroll-Ruderman and pion pole terms in the $\gamma N \rightarrow \pi N$ vertex. The three nucleon mechanisms discussed above, but driven by the background terms (non- $\Delta$ terms) in the $\gamma N \rightarrow \pi N$ amplitude, were not considered in Ref. [5] and the assumption was made that the proportion of three body to two body absorption would scale, as a function of energy, like the resonant pieces, in which case the three body terms would be negligible at the low photon energies where the nonresonant absorption dominates. However, the expected detail of the coming experiments and the existence of published work claiming a dominance of the $3 N$ mechanism around pion threshold $[8,9]$ call for a careful evaluation of this mechanism which we conduct in the next section.

The distinction between genuine and nongenuine three body absorption might look artificial or just a question of taste. This would be so if, in evaluating the amplitudes of the Feynman diagrams, there is an integration over the variable of the pion propagator which can become singular, as is the case in finite nuclei using nuclear wave functions, but not in nuclear matter. Let us clarify this. Take the amplitude corresponding to the photon absorption diagram below the dotted line in Fig. 3(e) and look at the pion in the serrated line closest to the photon. The pion propagator can become singular (pion on shell) and must be split into a principal part and a $\delta$ function. In finite nuclei the nucleons have a momentum distribution and one must integrate over the pion momentum when evaluating the amplitude, which then becomes well behaved. In nuclear matter, the nucleon states are plane waves; hence, the pion momentum is well defined for a given final state configuration and no momentum integration is required in the amplitude. Therefore, the cross section, obtained by squaring the amplitude and integrating over the final nucleon configurations, is infinite since it involves integrals of $\delta^{2}$ which are divergent. This might look like an absurd result but it is not. It is the exact measure of what one is evaluating there. Indeed, the physical meaning is illustrated in Ref. [20] and corresponds to the probability per unit length that there is a $\gamma N \rightarrow \pi N$ collision times the probability that the pion is absorbed by a pair of nucleons in nuclear matter integrated over the lifetime of the pion. Since the pion has become on shell (its propagator has zero width), it can live forever and the probability obtained is infinite. In finite nuclei, unlike in infinite nuclear matter, the real pion only meets pairs of nucleons in the finite volume of the nucleus and the probability is well behaved. In view of that it looks like the answer is clear: Evaluate these diagrams in finite nuclei. This is certainly a possibility and indeed some calculations are done in this way [6]. However, if one looks at the real situation in medium and heavy nuclei, where a pion can undergo two or three collisions before it is absorbed and each of the emitted nucleons can undergo a couple of collisions before they leave the nucleus [21], it becomes immediately clear that the Feynman diagrams associated with these processes in finite nuclei become technically prohibitive. Not in vain, these finite nuclei calculations are only available in very light nuclei like ${ }^{3} \mathrm{He}[6]$.

The realization of this complexity is what has forced people to tackle these problems with multiple ramifications using cascade methods, like the Monte Carlo simulation of Refs. $[7,21,22]$. The separation of the contribution where on shell pions appear in the final state is mandatory in such procedures. The methods are precisely based on probabilities of genuine processes where no on shell particles can appear as intermediate states in the corresponding amplitudes. Then the process which we have discussed, where a real pion appears after the $(\gamma, \pi)$ reaction and is subsequently absorbed, is naturally treated as a two-step process in the Monte Carlo simulation, hence the need to be excluded as a genuine process. Thus only processes with finite probability appear as input in the simulation. Furthermore, the two-step processes which had an infinite probability in infinite nuclear matter become finite, since the nuclear size is taken into account and when a pion leaves the nucleus it can no longer be scattered or absorbed by the nucleons.

The former discussion justifies the separation of genuine processes from the theoretical point of view. However, this is not the only justification since experimentally, by means of detailed analysis and selection of events in different regions of the Dalitz plot, one can separate approximately genuine from multistep processes. For instance, analysis of invariant masses of pairs of outgoing nucleons leads to distinctive peaks theoretically [20] and experimentally [23] if they come from the absorption of a real pion, and this can distinguish a $(\gamma, \pi)$ event followed by absorption of a real pion from a background of genuine three body absorption. 


\section{TWO AND THREE BODY ABSORPTION AROUND PION THRESHOLD}

By following the formalism of Refs. [16] and [18] we would like to evaluate the photon self-energy corresponding to the diagrams of Figs. 1(b) and 3 around the pion threshold. The results of Fig. 49 in Ref. [5] indicate that the $\Delta$-led terms account for less than $10 \%$ of the direct two body absorption cross section at this energy. Thus, we only consider here the Kroll-Ruderman and pion pole terms which are the dominant nonresonant terms in the $\gamma N \rightarrow \pi N$ amplitude [5] there. They only contribute for charged pions up to a small contribution in the $\gamma p \rightarrow \pi^{0} p$ case which we neglect. We also neglect terms of order $\mu / 2 M$ in the charged pion amplitudes, where $\mu$ and $M$ are the pion and nucleon mass, respectively. All these terms introduce corrections of order $(\mu / 2 M)^{2}$ in the final result of the photon self-energy and can thus be disregarded. With these simplifications the Kroll-Ruderman and pion pole terms are given by

$$
\begin{gathered}
-i t^{\mathrm{KR}}= \pm e \frac{f}{\mu} \sqrt{2} \vec{\sigma} \cdot \vec{\epsilon} F_{\pi}(k-q), \\
-i t^{\mathrm{PP}}= \pm e \frac{f}{\mu} 2 \sqrt{2} \vec{\epsilon} \cdot \vec{q} \vec{\sigma} \cdot(\vec{q}-\vec{k}) \frac{1}{(q-k)^{2}-\mu^{2}} F_{\pi}(k-q),
\end{gathered}
$$

where $f^{2} / 4 \pi=0.08, \vec{\epsilon}$ is the photon polarization vector (we work in the Coulomb gauge $\left.\epsilon^{0}=0, \vec{\epsilon} \vec{k}=0\right)$, and $F_{\pi}(k-q)$ is a monopole form factor with $\Lambda_{\pi}=1300 \mathrm{MeV}$ which we take from Ref. [24]. The $+(-)$ sign in Eq. (2) stands for the reaction $\gamma p \rightarrow \pi^{+} n\left(\gamma n \rightarrow \pi^{-} p\right)$.

In spin-isospin saturated nuclear matter, the photon selfenergy for the sum of $\pi^{+}$and $\pi^{-}$exchange in the diagrams of Figs. 1(b) and 3 is given by

$$
-i \Pi(k)=2 \int \frac{d^{4} q}{(2 \pi)^{4}} i \frac{1}{4} U_{N}(k-q)\left[i D_{0}(q)\right]^{2}\left[-i \Pi_{\pi}^{*}(q)\right] \overline{\sum_{\lambda}} \sum_{s_{i}} \sum_{s_{f}}(-i t)\left(-i t^{\dagger}\right),
$$

where $U_{N}(k-q)$ is the Lindhard function for $p h$ excitation, $D_{0}(q)$ the pion propagator, $t$ the $\gamma N \rightarrow \pi N$ amplitude, and $\Pi_{\pi}^{*}$ the pion self-energy. We sum over initial and final spins of the nucleons in $|t|^{2}$ and average over the photon polarization.

In order to obtain ImI we apply the Cutkosky rules [25] adapted to the present case,

$$
\begin{gathered}
\Pi(k) \rightarrow 2 i \operatorname{Im} \Pi(k), \\
U_{N}(k-q) \rightarrow 2 i \theta\left(k^{0}-q^{0}\right) \operatorname{Im} U_{N}(k-q), \\
\Pi_{\pi}^{*}(q) \rightarrow 2 i \theta\left(q^{0}\right) \operatorname{Im} \Pi_{\pi}^{*}(q),
\end{gathered}
$$

and we obtain

$$
\operatorname{Im} \Pi(k)=-\int \frac{d^{4} q}{(2 \pi)^{4}} \operatorname{Im} U_{N}(k-q) \theta\left(k^{0}-q^{0}\right) \theta\left(q^{0}\right) D_{0}(q)^{2} \operatorname{Im} \Pi_{\pi}^{*}(q) \overline{\sum_{\lambda}} \sum_{s_{i}} \sum_{s_{f}}|t|^{2}
$$

where the sum and average of $|t|^{2}$ is given by

$$
\overline{\sum_{\lambda}} \sum_{s_{i}} \sum_{s_{f}}|t|^{2}=F_{\pi}^{2}(k-q)|T|^{2}
$$

with

$$
|T|^{2}=4 e^{2} \frac{f^{2}}{\mu^{2}}\left\{P_{1}+2\left[\frac{1}{(q-k)^{2}-\mu^{2}}\right]^{2}(\vec{q}-\vec{k})^{2}\left[\vec{q}^{2}-\frac{(\vec{k} \cdot \vec{q})^{2}}{k^{2}}\right] P_{2}+2 \frac{1}{(q-k)^{2}-\mu^{2}}\left[\vec{q}^{2}-\frac{(\vec{k} \cdot \vec{q})^{2}}{k^{2}}\right] P_{3}\right\}
$$

and $P_{1}=P_{2}=P_{3}=1$.

The results of Eqs. (6) and (7) must still be corrected for effects of nuclear polarization and short range correlations. The polarization would account for the random phase approximation (RPA) diagrams depicted in Fig. 4. We follow here the same steps as in Ref. [5] to include both effects. The details needed for the present case are presented in the Appendix. When these corrrections are implemented Eq. (5) is replaced by 


$$
\begin{aligned}
\operatorname{Im} \Pi(k)= & -\int \frac{d^{4} q}{(2 \pi)^{4}} \operatorname{Im} U_{N}(k-q) \theta\left(k^{0}-q^{0}\right) \theta\left(q^{0}\right)\left[D_{0}(q) F_{\pi}(q) F_{\pi}(k-q)-\tilde{D}_{0}(q) \tilde{F}_{\pi}(q) \tilde{F}_{\pi}(k-q)\right]^{2} \\
& \times \frac{\left(f^{2} / \mu^{2}\right) \vec{q}^{2} \operatorname{Im} \bar{\Pi}_{\pi}^{*}(q)}{\left|1-V_{L}(q) \bar{\Pi}_{\pi}^{*}(q)\right|^{2}}|\bar{T}|^{2}
\end{aligned}
$$

where $\tilde{D}_{0}, \tilde{F}_{\pi}$ are the same functions $D_{0}, F_{\pi}$ but substituting $\vec{q}^{2}$ by $\vec{q}^{2}+q_{c}^{2}$ with $q_{c} \simeq 780 \mathrm{MeV}$. On the other hand, $|\bar{T}|^{2}$ is given in Eq. (7) taking the values of $P_{1}, P_{2}$, and $P_{3}$ defined in the Appendix. In Eq. (8), $V_{L}(q)$ stands for the longitudinal part of the spin-isospin interaction [see Eqs. (A2) and (A3) of the Appendix], and $\bar{\Pi}_{\pi}^{*}$ is related to $\Pi_{\pi}^{*}$ by means of

$$
\Pi_{\pi}^{*}(q)=\frac{f^{2}}{\mu^{2}} \vec{q}^{2} F_{\pi}^{2}(q) \bar{\Pi}_{\pi}^{*}(q),
$$

where $\Pi_{\pi}^{*}(q)$ is the irreducible self-energy in the sense that none of the diagrams it contains consists of pieces connected by $V_{L}$.

We now split $\bar{\Pi}_{\pi}^{*}$ in the numerator of Eq. (8) into its three components

$$
\bar{\Pi}_{\pi}^{*}(q)=\bar{\Pi}_{1 p 1 h}^{*}(q)+\bar{\Pi}_{\Delta h}^{*}(q)+\bar{\Pi}_{2 p 2 h}^{*}(q),
$$

corresponding to the coupling to $p h, \Delta h$, and $2 p 2 h$ excitations and defined in Eq. (A4) of the Appendix. Below the pion threshold we have $\operatorname{Im} \bar{\Pi}_{\Delta h}^{*}=0$, and then the contributions proportional to $\operatorname{Im} \bar{\Pi}_{1 p 1 h}^{*}$ or to $\operatorname{Im} \bar{\Pi}_{2 p 2 h}^{*}$ correspond to photon absorption by two nucleons and three nucleons, respectively, since one extra nucleon is implicit in $\operatorname{Im} U_{N}(k-q)$. As we have commented in former sections the two nucleon contribution is the one considered in Ref. [5] while the three nucleon contribution, obtained from the term proportional to $\operatorname{Im} \bar{\Pi}_{2 p 2 h}^{*}$, will be new.

We shall also extrapolate the results of this new channel to energies a bit above the pion threshold. Here one must deal also with the pion production channel. In this case, since $\operatorname{Im} \bar{\Pi}_{1 p 1 h}^{*}=0$ when the pion is on shell (no pion absorption by one nucleon), there is no admixture between the pion production mode and the two nucleon absorption channel. However, since $\operatorname{Im} \bar{\Pi}_{2 p 2 h}^{*} \neq 0$ when the pion can be on shell, the two modes are mixed and a separation must be made. In Ref. [7] this is done with the help of a Monte Carlo simulation, by letting a pion be produced according to the probability given by the diagram of Fig. 1(a), then propagate through the nucleus, and eventually be absorbed by two nucleons. As we mentioned, this gives rise to the phenomenon of indirect photon absorption. At very low pion energies we can take advantage of the fact that pions are little absorbed to make a simplified, yet realistic, calculation.

The first step is to add the pionic decay channel of Fig. 1(a) to our results of Eq. (8). This is easily done by means of

$$
\begin{aligned}
\operatorname{Im} \Pi^{(1 a)}(k)= & -\int \frac{d^{4} q}{(2 \pi)^{4}} \operatorname{Im} U_{N}(k-q) \theta\left(k^{0}-q^{0}\right) \theta\left(q^{0}\right) \\
& \times F_{\pi}^{2}(k-q) \operatorname{Im} D_{0}(q)|\bar{T}|^{2} .
\end{aligned}
$$

Although the sum of Eq. (8) and (11) seems to consist of a numerator with a $\delta$ function, from $\operatorname{Im} D_{0}(q)$, and a broad peak, from $\left|1-V_{L}(q) \bar{\Pi}_{\pi}^{*}(q)\right|^{-2}$, one can see, by summing analytically the two terms, that there is only a broad peak around the renormalized pion pole with a width related to $\operatorname{Im} \Pi_{2 p 2 h}^{*}$. The whole strength corresponds now to a pion in the medium. However, because of the spreading in momentum space of the pion strength, some regions of the spectrum cannot show up as a physical asymptotic pion because they would violate energy and momentum conservation, and they are forced to appear as $2 p 2 h$ excitations of the pionic mode. In order to evaluate the pion emission rate we take here the same prescription as in Ref. [18] which leads to realistic rates of pion emission in the $\Lambda$ nuclear decay. For this purpose we substitute, in Eq. (11),

$$
\operatorname{Im} D_{0} \rightarrow \operatorname{Im} D(q) \simeq-\pi \delta\left(\left(q^{0}\right)^{2}-\tilde{\omega}^{2}(q)\right),
$$

where $\tilde{\omega}(q)$ is the modified dispersion relation of the pion in the medium given by the solution of

$$
\tilde{\omega}^{2}(q)-\vec{q}^{2}-\mu^{2}-\Pi_{\pi}(\tilde{\omega}(q), q)=0
$$

The function $\Pi_{\pi}$,

$$
\Pi_{\pi}(q)=\frac{\Pi_{\pi}^{*}(q)}{1-\frac{f^{2}}{\mu^{2}} g^{\prime} \bar{\Pi}_{\pi}^{*}(q)}
$$

is the pion proper self-energy, which excludes those diagrams with pieces connected by one pion exchange (unlike $\Pi^{*}$ which excluded the diagrams connected by the full $V_{L}$, i.e., those connected by one pion exchange plus those connected by $g^{\prime}$, the parameter of the induced LandauMigdal interaction).

In order to extract the three body absorption contribution in the present case we evaluate the total ImI from the sum of Eqs. (8) and (11), subtract the two body absorption contribution obtained from Eq. (8) by keeping only $\operatorname{Im} \bar{\Pi}_{1 p 1 h}^{*}(q)$ in the numerator, and then subtract the pionic contribution with the prescription given above. The results are discussed in Sec. V.

\section{THREE BODY PHOTON ABSORPTION AT HIGHER ENERGIES}

As we go to higher energies, the $\Delta$ excitation becomes gradually more important and, at energies $300 \mathrm{MeV}$ $<E_{\gamma}<500 \mathrm{MeV}$, photon absorption is dominated by the $\Delta$ terms (see again Fig. 49 of Ref. [5]). In pion nucleus scattering the three body absorption has a maximum of about $60 \%$ of the total at energies $\omega_{\pi} \simeq 400 \mathrm{MeV}$. Here we should expect the same since at photon energies around $E_{\gamma}=400$ 
$\mathrm{MeV}$ the total absorption is $\Delta$ dominated in about $90 \%$. As a consequence we expect a curve for the ratio of direct two body photon absorption to the total direct absorption pretty much the same as the one given in Fig. 12 (solid line) of Ref. [2].

In Ref. [5] a detailed evaluation of the two body and three body absorption terms in the $\Delta$ region is done and hence we omit the details here. We simply summarize the most important elements in order to establish the connection with the evaluations done in the former section. The $\Delta$ excitation contribution to the photon self-energy is given by Eq. (44) of Ref. [5],

$$
\operatorname{Im} \Pi^{(\Delta)}(k)=-4 \int \frac{d^{3} p}{(2 \pi)^{3}} n(\vec{p})\left(\frac{f_{\gamma}}{\mu}\right)^{2} \frac{4}{9} \vec{k}_{\mathrm{c} . \mathrm{m} .}^{2} \frac{\frac{1}{2} \tilde{\Gamma}(k+p)+C_{Q}\left(\rho / \rho_{0}\right)^{\alpha}+C_{A 2}\left(\rho / \rho_{0}\right)^{\beta}+C_{A 3}\left(\rho / \rho_{0}\right)^{\gamma}}{\left|\sqrt{s}-M_{\Delta}+\frac{1}{2} i \tilde{\Gamma}(k+p)-\Sigma_{\Delta}(k+p)\right|^{2}}
$$

where $f_{\gamma}=0.12$ is the $\gamma N \Delta$ coupling constant, $\vec{p}$ is the momentum of the nucleon in the Fermi sea, $k_{\text {c.m. }}$ the photon momentum in the $\gamma N$ center of mass system, $\tilde{\Gamma}$ the Pauli corrected $\Delta$ width, and $C_{Q}, C_{A 2}, C_{A 3}$ coefficients of the terms of the imaginary part of the $\Delta$ self-energy related to the $(\gamma, \pi)$ channel, two body, and three body absorption, respectively. Analytical parametrizations of these coefficients can be found in Ref. [26].

Equation (15) corresponds actually to the diagrams of Fig. 2 , where iterations (in the Dyson sense) of the $\Delta$ self-energy insertions in the figure are implicitly assumed. This is automatically done by adding the proper self-energy to the $\Delta$ mass in the $\Delta$ propagator. The imaginary part of the $\Delta$ propagator is the fraction appearing in Eq. (15). The sources of $\operatorname{Im} \Sigma_{\Delta}$ shown in the numerator can be traced back to the contribution of the diagrams in Fig. 2. The term with $\tilde{\Gamma}$ is associated with diagram (a) $[(\gamma, \pi)]$, the term with $C_{Q}$ to the cut in diagram (c) [correction term to $(\gamma, \pi)]$, the term with $C_{A 2}$ to diagram (b) (two body absorption), and the term with $C_{A 3}$ to diagram (e) (three body absorption). Diagram (d) ac-

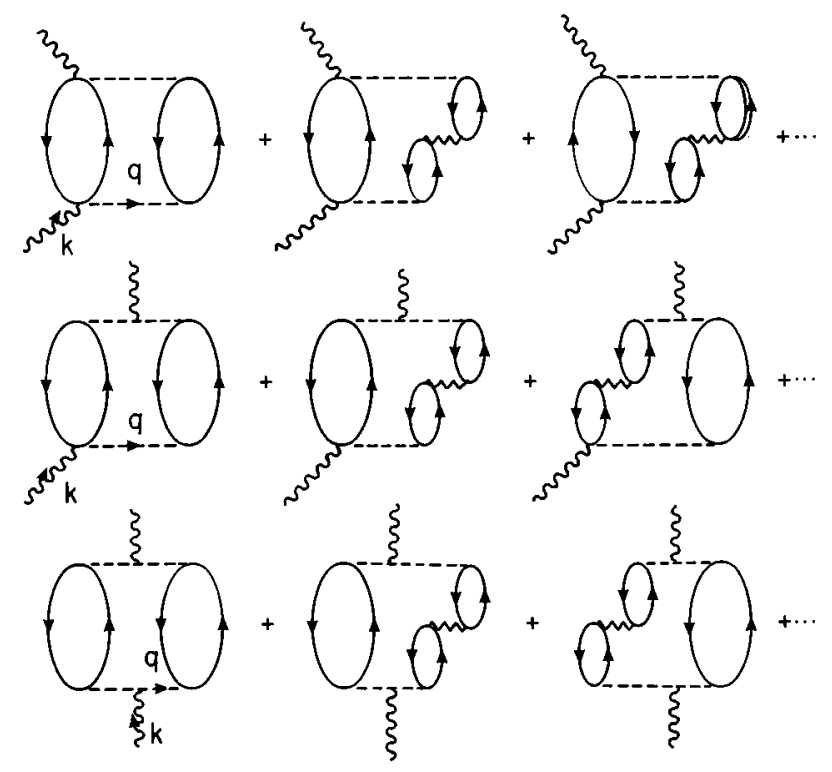

FIG. 4. Photon self-energy diagrams, with Kroll-Ruderman and pion pole terms in the $\gamma N \rightarrow \pi N$ amplitude, showing the medium polarization through RPA $p h$ and $\Delta h$ excitations induced by the pion. counts for corrections to $(\gamma, \pi)$ and is included in the $C_{Q}$ term, and there is also a term included in $C_{A 3}$ which corresponds to Fig. 5.

For completeness we should also mention that in Ref. [5] a new source of genuine three body absorption is calculated tied to the $\gamma N \rightarrow \pi \pi N$ channel, instead of the $\gamma N \rightarrow \pi N$ to which the former pieces are tied via exchange current mechanisms. The corresponding diagram is shown in Fig. 6. It contributes only appreciably at energies above the $\Delta$ region. For instance, at $E_{\gamma}=450 \mathrm{MeV}$ it contributes about $10-$ $15 \%$ of the cross section, which is a sizable fraction of the direct photon absorption cross section.

The results for the total $\gamma$ cross section and direct photon absorption can be found in Ref. [5]. In the next section we show the splitting of this cross section in two and three body contributions.

\section{RESULTS AND DISCUSSION}

Our results for direct two and three nucleon photon absorption are shown in Fig. 7 as a function of the photon energy for two different nuclei, ${ }^{12} \mathrm{C}$ (solid line) and ${ }^{208} \mathrm{~Pb}$ (dashed line). We observe that the two body absorption results are consistent with those obtained in Ref. [5]. The three

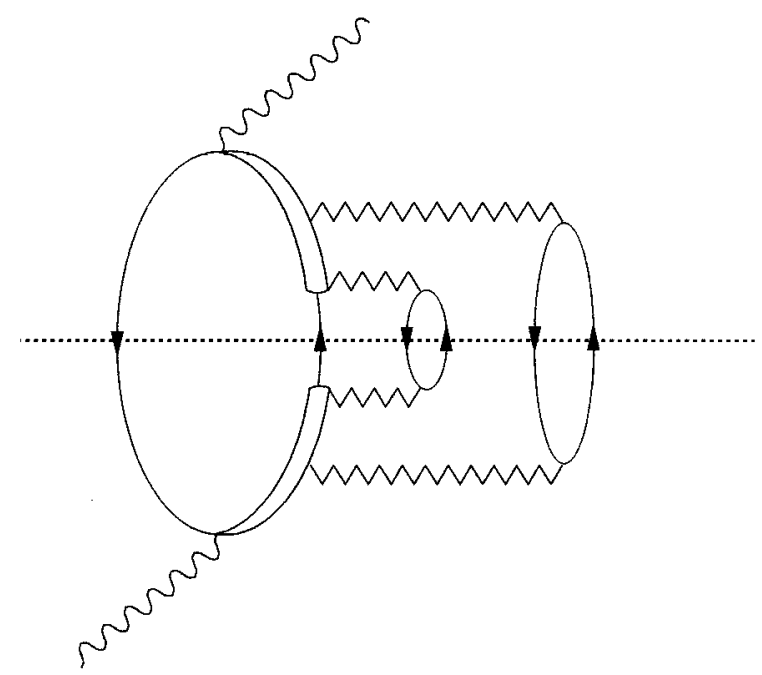

FIG. 5. Three body absorption diagram also included in Eq. 


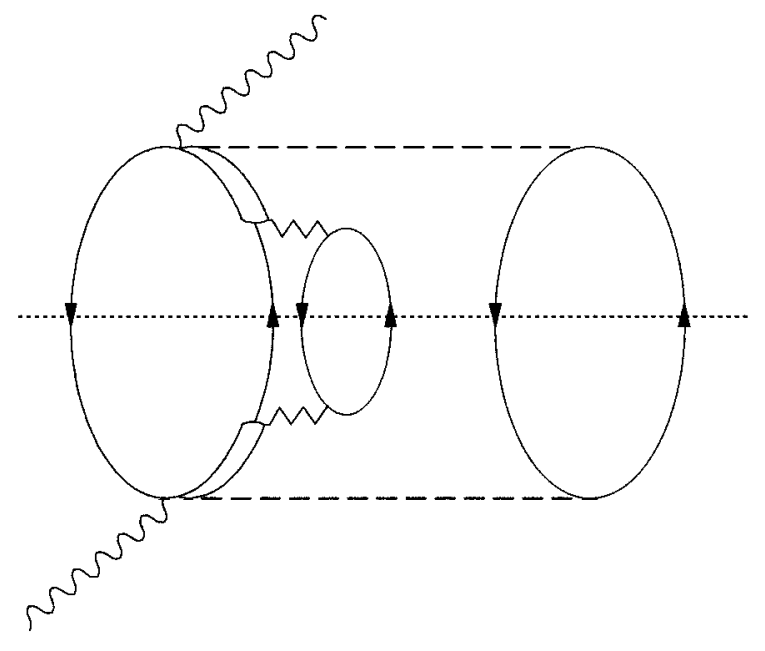

FIG. 6. Three body absorption diagram considered in Ref. [5] and which provides contribution above the $\Delta$ resonance region.

nucleon absorption cross section is very small, of the order of $2-12 \%$ of the two nucleon absorption channel in the range $100 \mathrm{MeV}<E_{\gamma}<200 \mathrm{MeV}$. These results show that photon absorption around pion threshold is essentially a two body process (leaving apart final state interaction of the nucleons) and strengthens the results obtained in Refs. [5] and [7].

One can wonder why these results are so much smaller than those obtained in Ref. [8]. For this purpose let us recall that several approximations were done in Ref. [8], which necessarily lead to a larger three nucleon absorption rate. On the one hand a constant density $\rho=\rho_{0}$ was used in Ref. [8], while we use here the local density approximation. Since the three body absorption terms are roughly of order $\rho^{3}$, one can see from Eq. (1) that $\sigma_{A 3} / A$ is proportional to $\int d^{3} r \rho^{3}(r) / A \equiv C \rho_{0}^{2}$. Using a $r$-dependent density we obtain $C=0.32,0.36$, and 0.66 for ${ }^{12} \mathrm{C},{ }^{16} \mathrm{O}$, and ${ }^{208} \mathrm{~Pb}$, respectively, while $C=1$ for a sphere of constant density $\rho_{0}$. On the other hand a pion self-energy independent of $q^{0}$ is also employed in Ref. [8]. This does not provide the necessary off shell dependence of the self-energy needed for the present

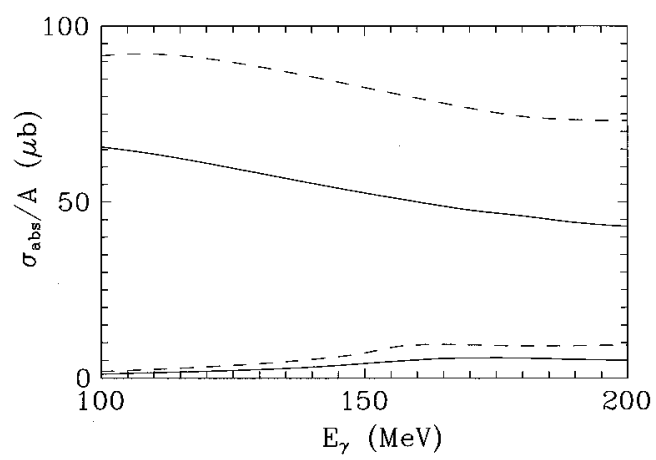

FIG. 7. $\sigma_{\mathrm{abs}} / A$ as a function of the photon energy for direct two body and three body absorption. Upper two lines: two body absorption. Lower two lines: three body absorption. Dashed lines are for ${ }^{208} \mathrm{~Pb}$ and solid lines for ${ }^{12} \mathrm{C}$. Only the Kroll-Ruderman and pion pole terms are included in the $\gamma N \rightarrow \pi N$ vertex.

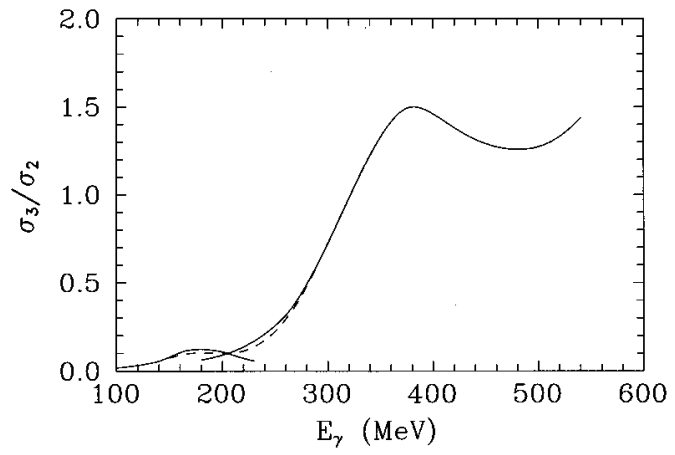

FIG. 8. $\sigma_{A 3} / \sigma_{A 2}$ : ratio of three to two body direct photon absorption in ${ }^{12} \mathrm{C}$ as a function of the photon energy. See explanation in the text.

problem. As was shown in Ref. [18], neglecting this leads again to an overestimate of the rate. Furthermore, the nuclear polarization effects discussed here also lead to a reduction as was shown in Ref. [5]. The apparent good results compared to the data obtained in Ref. [8], using only the three body absorption channel, are not surprising in view of the previous discussion and the fact that there was a cutoff parameter in Ref. [8] which was adjusted to fit the data.

In Fig. 8 we show the results for the ratio $\sigma_{A 3} / \sigma_{A 2}$ of three to two body direct photon absorption in ${ }^{12} \mathrm{C}$ as a function of the photon energy. A very similar ratio is obtained for heavier nuclei like ${ }^{208} \mathrm{~Pb}$.

The curves in Fig. 8 are obtained as follows. The line spanning from $E_{\gamma}=100 \mathrm{MeV}$ to $230 \mathrm{MeV}$ corresponds to a calculation in which only the Kroll-Ruderman and pion pole terms are included in the $\gamma N \rightarrow \pi N$ amplitude. These are the dominant ones for energies around pion threshold. The line spanning from $E_{\gamma}=180 \mathrm{MeV}$ up to $540 \mathrm{MeV}$ corresponds to photon absorption led by the $\Delta$ excitation terms alone which are calculated from Eq. (15). These two curves are dominant at low $(<150 \mathrm{MeV})$ and high energies $(>300 \mathrm{MeV})$, respectively, and, as we see in the figure, they cross each other around $E_{\gamma}=210 \mathrm{MeV}$ which is also a transition point for the dominance of the two types of mechanisms. A calculation containing all terms together would merge the results of Fig. 8 into a continuous curve which would show up as a smooth interpolation between the two curves around the crossing region. This is indicated by the dashed line, which is merely a guide to the eye. Inclusion of the extra terms considered in Ref. [5] at higher energies does not change the figure much, with the background terms adding to two body absorption and the contribution from Fig. 6 to three body absorption. Hence, the line in Fig. 8 spanning from $E_{\gamma}=100 \mathrm{MeV}$ to 550 $\mathrm{MeV}$ gives a fair account of the predictions of the model of Ref. [5] complemented with the background three body absorption terms introduced in the present work. We can summarize our findings by saying that two body absorption is dominant at low energies but from energies $E_{\gamma} \simeq 400 \mathrm{MeV}$ on the three body contribution is sizable and comparable, even bigger, than the two body one, as was also found for three body pion absorption [2].

\section{CONCLUSIONS}

In view of theoretical claims [8] that photon absorption at energies around pion threshold is dominated by three 
nucleon absorption and the contradiction with other theoretical claims [5] that it is given by two body absorption, we carried a thorough investigation of the three nucleon channel incorporating all the theoretical ingredients that have proved relevant in recent studies of photon nucleus interaction. The experience gained in the study of one nucleon and two nucleon induced $\Lambda$ decay $[16,18]$, which is analogous to the present problem, was of much use. The results obtained here give photon absorption rates by three nucleons which are of the order of $10 \%$ or less than the two body one around pion threshold and stresses the two body dominance of the photon absorption mechanisms at these energies. The large results obtained in Ref. [8] were traced back to different approximations used there, all of which magnified the relevance of the three body channel. At large photon energies dominated by $\Delta$ excitation the situation is different and, analogously to the results obtained in pion absorption, which uses the same input for the $\Delta$ self-energy as the study of photon absorption in Ref. [5], one finds here that the ratio of three body absorption to two body absorption increases smoothly from a few percent at energies around pion threshold up to a ratio close to 1.5 at photon energies around $400 \mathrm{MeV}$, or equivalently, three body absorption represents there about $60 \%$ of the total absorption.

We expect that the coming photon experiments will shed light on this issue much as the experiments in Ref. [1] have shed much light on the question of the number of nucleons involved in pion absorption.

\section{ACKNOWLEDGMENTS}

We wish to acknowledge financial support from the EU under the program of Human Capital and Mobility Contract No. CHRX-CT 93-0323. This work has also been partly supported by CICYT Contracts Nos. PB 92-0761 and AEN 931205.

\section{APPENDIX: POLARIZATION AND CORRELATION CORRECTIONS}

The nuclear polarization effects proved to be important in Ref. [5]. We show here how they are incorporated in the terms which we are considering. Nuclear polarization effects are taken into account by summing up the RPA diagrams implicit in Fig. 4. We follow here the steps of Sec. 9 of Ref. [5]. On the one hand we must substitute

$$
\operatorname{Im}_{\pi}^{*}(q) \rightarrow \frac{\operatorname{Im} \Pi_{\pi}^{*}(q)}{\left|1-V_{L}(q) \bar{\Pi}_{\pi}^{*}(q)\right|^{2}}
$$

where $V_{L}(q)$ is the longitudinal part of the $p h$ interaction,

$$
V_{p h} \equiv\left\{V_{L}(q) \hat{q}_{i} \hat{q}_{j}+V_{T}(q)\left(\delta_{i j}-\hat{q}_{i} \hat{q}_{j}\right)\right\}
$$

with

$$
\begin{gathered}
V_{L}(q)=\frac{f^{2}}{\mu^{2}}\left[\vec{q}^{2} F_{\pi}^{2}(q) D_{0}(q)+g^{\prime}\right], \\
V_{T}(q)=\frac{f^{2}}{\mu^{2}}\left[\vec{q}^{2} F_{\rho}^{2}(q) D_{\rho}(q) C_{\rho}+g^{\prime}\right],
\end{gathered}
$$

$g^{\prime}=0.6, D_{\rho}$ the $\rho$ propagator, $C_{\rho}=2$, and $F_{\rho}$ the monopole $\rho$ form factor with $\Lambda_{\rho}=2500 \mathrm{MeV}$ [18]. Another version using $C_{\rho}=3.96$ and $\Lambda_{\rho}=1400 \mathrm{MeV}$ [24] leads basically to the same results. The function $\bar{\Pi}_{\pi}^{*}$, related to $\Pi_{\pi}^{*}$ by means of Eq. (9), contains, as shown in Eq. (10), $p h, \Delta h$, and $2 p 2 h$ excitation terms given by

$$
\begin{gathered}
\bar{\Pi}_{1 p 1 h}^{*}(q)=U_{N}(q), \\
\bar{\Pi}_{\Delta h}^{*}(q)=U_{\Delta}(q), \\
\bar{\Pi}_{2 p 2 h}^{*}(q)=-4 \pi \operatorname{Re} C_{0}^{*} \rho^{2}\left(\frac{f^{2}}{\mu^{2}}\right)^{-1} \\
-i 4 \pi \operatorname{Im} C_{0}^{*} \rho_{\mathrm{eff}}^{2} \frac{P H\left(q^{0}, q, \rho\right)}{P H\left(\mu, 0, \rho_{\mathrm{eff}}\right)}\left(\frac{f^{2}}{\mu^{2}}\right)^{-1},
\end{gathered}
$$

where $U_{N}, U_{\Delta}$ are the ordinary Lindhard functions for $p h$ or $\Delta h$ excitation [27] with the normalization of the appendix of Ref. [28]. We use the value $C_{0}^{*}=(0.105+i 0.096) \mu^{-6}$ [18]. The function $P H$ takes into account the phase space available for real $2 p 2 h$ excitation with incoming momentum $q^{0}, \vec{q}$ in nuclear matter at density $\rho$. Explicit expressions for $P H$ can be found in Eqs. (27)-(31) of Ref. [18].

What one has done in Eq. (A4) is to take the empirical input for $\Pi_{2 p 2 h}^{*}$ from pionic atoms and extend it to off shell situations. This is done by multiplying the on shell values by the ratio of phase space for $2 p 2 h$, at the appropriate values of $q^{0}, q$, and $\rho$, to the one for the case of pionic atoms, namely $q^{0}=\mu, q=0$, and $\rho_{\text {eff }}=0.75 \rho_{0}$ [29].

On the other hand, Fig. 4 contains also the polarization of the $p h$ excitation on the left of the diagram of Fig. 1(b). These corrections must also be implemented in the diagram of Fig. 3. This affects in a different way the three contributions to $|T|^{2}$ in Eq. (7). The first term corresponds to the square of the Kroll-Ruderman term while the other two correspond to the square of the pion pole term and the interference of the two terms. The polarization can be taken into account modifying the terms in Eq. (7) as done in Sec. 9 of Ref. [5]. Each term is multiplied by the factor $P_{i}$, with $i=1,2,3$, respectively, where

$$
\begin{gathered}
P_{1} \equiv \frac{1}{\left|1-V_{L}(k-q) \bar{\Pi}^{*}(k-q)\right|^{2}} \frac{\vec{q}^{2}}{(\vec{k}-\vec{q})^{2}} \frac{1}{2}\left(1-\cos ^{2} \theta\right)+\frac{1}{\left|1-V_{T}(k-q) \bar{\Pi}^{*}(k-q)\right|^{2}}\left[1-\frac{\vec{q}^{2}}{(\vec{k}-\vec{q})^{2}} \frac{1}{2}\left(1-\cos ^{2} \theta\right)\right], \\
P_{2} \equiv P_{3} \equiv \frac{1}{\left|1-V_{L}(k-q) \bar{\Pi}^{*}(k-q)\right|^{2}},
\end{gathered}
$$


and $\theta$ is the angle between $\vec{k}$ and $\vec{q}$.

The difference in the three terms appears because the first term is a mixture of spin longitudinal and transverse parts, while the other two are of pure spin longitudinal nature.

The polarization takes into account long range correlations. One must still correct for the effect of short range correlations and this is done again following the steps of Ref. [5] (Appendix D). We use here a simplified version of this latter work, which leads practically to the same results, and consists in replacing

$$
D_{0}(q) F_{\pi}(q) F_{\pi}(k-q) \rightarrow D_{0}(q) F_{\pi}(q) F_{\pi}(k-q)-\tilde{D}_{0}(q) \tilde{F}_{\pi}(q) \tilde{F}_{\pi}(k-q),
$$

where $\tilde{D}_{0}, \tilde{F}_{\pi}$, are the same functions $D_{0}, F_{\pi}$ but substituting $\vec{q}^{2}$ by $\vec{q}^{2}+q_{c}^{2}$, where $q_{c} \simeq 780 \mathrm{MeV}$ is the inverse of a typical nuclear correlation distance [30].

[1] (a) G. Backenstoss et al., Phys. Rev. Lett. 55, 2782 (1985); (b) R. Tacik et al., Phys. Rev. C 40, 256 (1989); (c) H. J. Weyer, Phys. Rep. 195, 295 (1990); (d) D. Mack, Nucl. Phys. A527, 451c (1991); (e) P. Weber et al., ibid. A534, 542 (1991); (f) R. D. Ransome et al., Phys. Rev. C 45, 509 (1992).

[2] E. Oset, Y. Futami, and H. Toki, Nucl. Phys. A448, 597 (1986).

[3] E. Oset and D. Strottman, Phys. Rev. C 42, 2454 (1990).

[4] K. Masutani and K. Yazaki, Phys. Lett. 104B, 1 (1981); Nucl. Phys. A407, 309 (1983).

[5] R. C. Carrasco and E. Oset, Nucl. Phys. A536, 445 (1992).

[6] J. M. Laget, Phys. Rev. C 38, 2993 (1988).

[7] R. C. Carrasco, E. Oset, and L. L. Salcedo, Nucl. Phys. A541, 585 (1992).

[8] P. Christillin, J. Phys. G 10, L65 (1984).

[9] P. Chistillin, J. Phys. G 11, 795 (1985); Phys. Rep. 190, 63 (1990).

[10] P. Carlos et al., Nucl. Phys. A431, 573 (1984).

[11] L. Ghedira, Ph.D. thesis, Université de Paris Süd, 1984.

[12] P. Ahrens et al., Nucl. Phys. A490, 655 (1988).

[13] W. Bertozzi, in Proceedings of the Workshop on Two Nucleon Emission Reactions, edited by O. Benhar and A. Fabrocini (ETS Editrice, Pisa, 1990), p. 25; L.B. Weinstein et al., Phys. Rev. Lett. 64, 1646 (1990).

[14] N. D'Hose et al., Phys. Rev. Lett 63, 856 (1989).

[15] P. Grabmayr (private communication); N. Rodning (private communication); D. Brandford (private communication); A. Sandorfi (private communication); D. MacGregor (private communication); G. E. Cross et al., Glasgow University report, 1995.

[16] W. M. Alberico, A. de Pace, M. Ericson, and A. Molinari, Phys. Lett. B 256, 134 (1991).

[17] J. J. Szymansky et al., Phys. Rev. C 43, 849 (1991).

[18] A. Ramos, E. Oset, and L. L. Salcedo, Phys. Rev. C 50, 2314 (1994).

[19] I. Blomqvist and J. M. Laget, Nucl. Phys. A280, 405 (1977).

[20] L. L. Salcedo, E. Oset, D. Strottman, and E. Hernández, Phys. Lett. B 208, 339 (1988).

[21] L. L. Salcedo, E. Oset, M. J. Vicente-Vacas, and C. GarcíaRecio, Nucl. Phys. A484, 557 (1988).

[22] R. C. Carrasco, M. J. Vicente-Vacas, and E. Oset, Nucl. Phys. A570, 701 (1994).

[23] J. Köhler, in Mesons and Light Nuclei '95, edited by J. Adam, J. Dobes, R. Mach, M. Sotona, and J. Dolejsi [Few-Body Systems Suppl. 9 (in press)].

[24] R. Machleidt, K. Holinde, and Ch. Elster, Phys. Rep. 149, 1 (1987).

[25] G. Itzykson and J. B. Zuber, Quantum Field Theory (McGrawHill, New York, 1980).

[26] E. Oset and L.L. Salcedo, Nucl. Phys. A468, 631 (1987).

[27] A. L. Fetter and J. D. Walecka, Quantum Theory of Many Particle Systems (McGraw-Hill, New York, 1971).

[28] E. Oset, P. Fernández de Córdoba, L. L. Salcedo, and R. Brockmann, Phys. Rep. 89, 79 (1990).

[29] R. Seki and K. Masutani, Phys. Rev. C 27, 2799 (1983).

[30] E. Oset and W. Weise, Nucl. Phys. A319, 477 (1979). 